\title{
APPLICATION OF SLIDING WINDOWS TO SPELLING ERROR DETECTION IN MEDICAL DIAGNOSIS
}

\author{
Sutat Gammanee ${ }^{1}$ and Sunantha Sodsee ${ }^{2}$ \\ ${ }^{I}$ Doctoral student \\ ${ }^{2}$ Assistant Professor \\ King Mongkut's University of Technology North Bangkok, Faculty of Information Technology and Digital Innovation, \\ King Mongkut's University of Technology North Bangkok, Bangkok, Thailand
}

\begin{abstract}
Presently, problematic issues relative to health and public health in Thailand are underlined by an insufficient number of medical personnel. However, self-diagnosis, herein, is another option to help the mentioned limitation by identifying and monitoring unusual health conditions by oneself. To gain the diagnosis, history of medical data based on symptoms and disease of patients are needed significantly. In fact, especially in Thailand, medical data is recorded, not only presented by, such as: numeric data, images, but also describing by words or phrases. Then, the errors of medical data collection may be occurred by incorrect data or spelling errors of describing.

To enhance the performance of self-diagnosis, in this paper, the medical data in describing by medical words is focused. The detection of spelling errors and measuring similar words are coped with the proposed sliding window based methods, which are 1) sliding window based two-word inspection by once sliding method 2) sliding window based two-word inspection by initial word fixing method, and 3) sliding window based window size adjusting method.

For evaluating the proposed work, the example medical data (without patient information) is gained from the public hospital in Thailand. The results presented that the sliding window based two-word inspection by initial word fixing method presented the great accuracy of spelling errors detection about $80 \%$ in general words and $60 \%$ of similar words measurement in medical terms.
\end{abstract}

\section{KEYWORDS}

Self-Diagnosis, Spelling Errors Detection, Sliding Windows

\section{INTRODUCTION}

Problematic issues in public health have currently become a major consideration, and a number of studies have been conducted in attempts to solve the problems. One of the underlined causes is an insufficient number of medical professionals - the ratio of medical personnel population was reported at 1:412 (Sawaengdee, 2017). Accordingly, a policy had been launched by government in order to promote self-care practices and reduce medical visits (Phalasuek, Thanomchayathawatch, \& Songloed, 2018) by regularly doing exercises and performing self-diagnosis.

Self-diagnosis is the process of identifying and monitoring unusual health conditions by oneself for further medical treatments or basic self-care to prevent risk conditions and maintain the best curing conditions before the health problems become incurable. Currently, people are suffering from hard-working routines, and physical examination is often regarded as a very time-consuming task. As a result, a large number of patients decided to have medical visits when their health problems become incurable illness. Therefore, self-diagnosis could assist in reducing health problems, medical visits, and government budget in health promotion.

According to (Gammanee \& Sodsee, 2018), raw data obtained by medical diagnosis had been categorized and gained two groups of patients - patients with normal conditions and patients with risk conditions. After categorization, the data of patients with risk conditions were analysed using the process of self-diagnosis performed by medical personnel.

The problem in this research is to diagnose the disease, based on the analysis of the medical diagnosis, Generally, medical diagnosis is recorded in forms of messages describing symptoms using medical terms which are uneasy for recording and spelling. Thus, spelling errors may occur during the recording process that may 
lead to false diagnosis e.g., 'Dysentery is the symptom of intestinal inflammation.', 'Hyperglycemia is the condition with too much glucose is blood circulation.', and 'Abdominal Discomfort is the symptom of pain that occurs between the chest and pelvic regions.' As seen from the above examples, a number of medical terms are similar in terms of spelling and could replace other words containing similar meanings — only written forms and word categories are different e.g., 'Abdomen' and 'Abdominal', both words refer to the symptom of stomachache and flatulence; as analyzed by machine learning, the words were interpreted as different words. Whereas, experts confirm similarity and interchangeability of both words. As a result, the analysis could be incorrect and lead to failure in medical treatment. The researchers propose of the concept of how Sliding Windows came to the application to group the vocabulary before processing it with a machine as the concept frame in Figure 1.

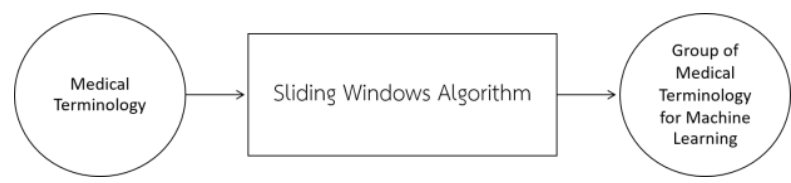

Figure 1. Appling Sliding Windows to Solve Research Problems

The concept of Sliding Windows is the process used in controlling accurate data-transmission with protocol between the sender and receiver by determining relative window sizes, sliding both sides at once, and detecting data until the process is complete. In this case, data detection of Sliding Windows is the pros that could handle with two similar meaning words. Sliding Windows was therefore adopted in this study to detect similarity of medical terms in order to categorize similar words into the same group. The researchers introduced the concept of sliding windows to the design to detect a similar word from a medical diagnosis to group the medical term that can be in the same group. In order to make the diagnosis more accurate. In addition, the benefits of this research can also learn similar words to solve problems with your doctor's information to correct them.

\section{RELATED WORKS}

\subsection{Sliding Windows}

Sliding Windows is the concept used in designing with arrays that is mostly selected for inspecting data-transmission between the sender and receiver by determining window sizes starting from the first point and continuing to slide to the right by point-by-point. The technique has been applied in various tasks. As seen in Figure 2:

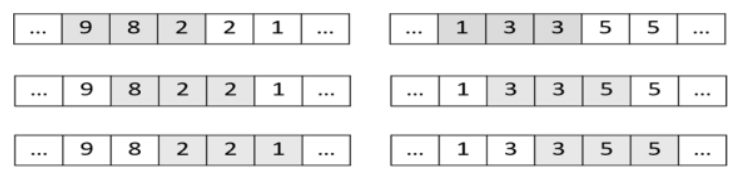

Figure 2. Sliding Windows for data accuracy

According to Figure 2, Sliding Windows is used as a protocol for inspecting data accuracy within the network (Moore \& Papagiannaki, 2005), it is additionally adopted to identify a variable of arrays (Kohler, Gulati, Cao, Hart, \& Ludascher, 2012).

\subsubsection{Sliding Windows for Data Communication}

Sliding Windows is used in data communication with multiple sets of data transmission to the receiver according to specified sizes for data inspection of both sides. The technique could assist in reducing memory storage use and maximizing data transmission speed. Besides, it is considered as a type of data transmission.

Some advantages of data transmission using Sliding Windows are multiple frames of data transmission and data inspect ability due to similar window sizes. 


\subsubsection{Sliding windows for Arrays}

Sliding windows is used with arrays for accurate inspection of data steaming by determining suitable window sizes and sliding both sides at once. Besides, it could assist in arranging data and determining maximum values of arrays.

\subsection{Commonly Misspelled Words in English}

Commonly, misspelled words are seen in English writing. According to (Flor, 2012) (Mackay, 1969) (Healy, 1981), a number of misspelled words were retrieved and demonstrated as follows:

\subsubsection{Correctly Spelled-Words Plus Additional Character}

Words are correctly spelled, but an additional character (s) is inserted in. There are 3 patterns of the case - an additional character (s) at the final position e.g., computer - computers, an additional character (s) at the initial position e.g., computer - computer, and an additional character (s) at the middle position e.g., computer computer.

Words are correctly spelled, but an additional character (s) is inserted in. There are 3 patterns of the casean additional character (s) at the final position e.g., computer - computers, an additional character (s) at the initial position e.g., computer - computer, and an additional character (s) at the middle position e.g., computer - computer.

\subsubsection{Partially Misspelled-Words}

Words are incorrectly spelled by a wrong character (s). There are 2 patterns of the case - a wrong character (s) spelling, but containing the correct number of characters, e.g., computer - comtuter, and a wrong character (s) spelling and containing an incorrect number of characters e.g., computer -comtter.

\subsubsection{Correctly Spelled-Words with Missing Characters}

Words are correctly spelled with a missing character (s). There are 2 patterns of the case- -missing a character (s) at the final position, e.g., computer - compute, and missing a character (s) at the initial position e.g., computer - omputer.

\section{EVALUATING ALGORITHM EFFICIENCY}

This study adopted Sliding Windows to detect of word similarity with an application of 3 Sliding Windows methods as follows:

\subsection{Sliding Window Based Two-Word Inspection by Once Sliding Method}

This method slides windows with 3 characters starting from left to right at once, then detects both windows for similarity of contained characters. As seen in Figure 3:

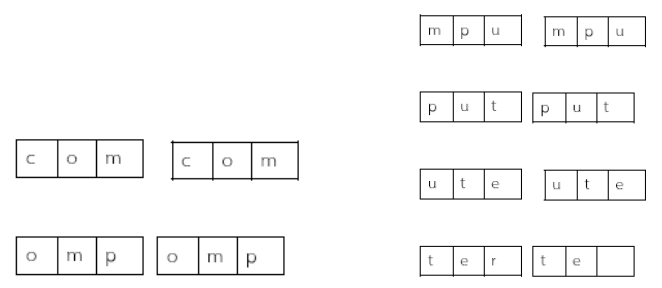

Figure 3. The working of Two-word Inspection by Sliding at Once method

An evaluation was conducted by counting points of inspection of both windows-correct point (similar spelling) as 1 and incorrect point (different spelling) as 0 , then summarized all points, divided by a full point, and multiplied by 100 to determine percentage of correctness. 


$$
\mathrm{C}(\mathrm{st} 1, \mathrm{st} 2)=\left\{\begin{array}{c}
1, \text { When same all string } \\
0, \text { When not same all string }
\end{array}\right.
$$$$
\text { Percentage }=\frac{\sum_{\mathrm{i}=1}^{\mathrm{m}} \mathrm{c}(\mathrm{st} 1[\mathrm{i}, \mathrm{i}+1, \mathrm{i}+2], \mathrm{st} 2[\mathrm{i}, \mathrm{i}+1, \mathrm{i}+2])}{\mathrm{i}} \times 100
$$

By $\mathrm{Ci}$ refers to function for checking same character i refers to position of string st1 refers to first string to compare st 2 refers to second string to compare $\mathrm{m}$ refers to the number of string length

\subsection{Sliding Window Based Two-Word Inspection by Initial Word Fixing}

This method slides windows by remaining an initial word unmoved and moves the rest, then moves the initial word by point-by-point and the rest until all characters have been moved, then inspects for correctness. As seen in Figure 4: Inspection of 'comp' and 'comt'.

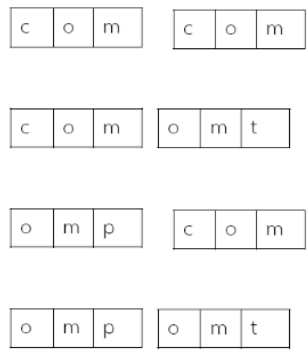

Figure 4. The working of Two-word Inspection by Fixing Initial Word method

$$
\mathrm{C}(\mathrm{st} 1, \mathrm{st} 2)=\left\{\begin{array}{cl}
1, \text { When same all string } & \text { Percentage }=\frac{\mathrm{p}}{(\mathrm{m}-3) \times 3} \times 100 \\
0, \text { When not same all string } & \mathrm{P}=\sum_{\mathrm{i}}^{\mathrm{m}-3} \mathrm{C}\left(\mathrm{st} 1[\mathrm{i}, \mathrm{i}+1, \mathrm{i}+2], \sum_{\mathrm{j}}^{\mathrm{m}-3}{ }_{\mathrm{st}} 2(\mathrm{j}, \mathrm{j}+1, \mathrm{j}+2)\right)
\end{array}\right.
$$

By $\mathrm{Ci}$ refers to function for checking same character

i refers to position of string

st 1 refers to first string to compare

st2 refers to second string to compare

$P$ refers to similarity score

$\mathrm{m}$ refers to the number of string length

\subsection{Sliding Window Based Window Size Adjusting}

An adjustment of window size was performed by increasing the window of characters from 1 to 2 until the window size contains the same size of word characters. Then, the first set of word is searched in comparison to the second word until the window size contains the same size of word characters. As seen in Figure 5. 


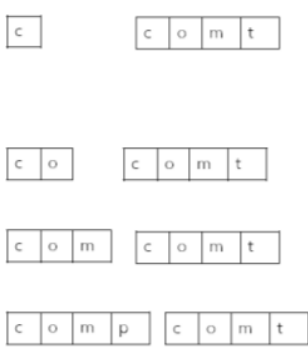

Figure 5. The working of Adjusting Window Sizes method

$$
\mathrm{C}(\mathrm{st} 1, \mathrm{st} 2)=\left\{\begin{array}{c}
1, \text { When same all string } \\
0 \text {, When not same all string }
\end{array} \quad \text { Percentage }=\frac{\max \sum_{\mathrm{i}}^{\mathrm{m}} \mathrm{c}\left(\mathrm{st} 1[\mathrm{i}], \sum_{\mathrm{j}}^{\mathrm{m} s t} 2[\mathrm{j}, \ldots, \mathrm{m})\right)}{\mathrm{m}} \times 100\right.
$$

By $\mathrm{Ci}$ refers to function for checking same character

i refers to position of string

st1 refers to first string to compare

st2 refers to second string to compare

$\mathrm{m}$ refers to the number of string length

Counting points was performed on each evaluation task and calculated in forms of percentage. The percentage was calculated using the number of characters divided by max number character, and multiplied by 100 - the probability $(\mathrm{P})$ was equivalent to the maximum value of percentage.

\section{EXPERIMENT AND RESULTS}

To evaluate the proposed sliding window based methods, the tests are divided in to two major parts, which are 1) spelling error detection in general words consisting of basic vocabulary and misspelled words retrieved from research (Fitzgerald, 1932), and 2) measuring similar word in medical terms.

\subsection{Spelling Error Detection in General Words}

\subsubsection{Correctly Spelled-Words Plus Additional Character}

In this subsection, the word "computer" is selected to test the proposed work by concerning major seven patterns of error (Flor, 2012). The results presented in Table 1 show that three proposed methods presented excellent detecting of spelling error on Pattern 1 and Pattern 6, which the accuracies are greater than $80 \%$.

On the other hand, the method 1: sliding window based two-word inspection by once sliding method could not detect the error on Pattern 2 and Pattern 7 appropriately, which accuracies are $4.76 \%$ and $0 \%$, respectively. Method 2: sliding window based two-word inspection by initial word fixing method showed accuracies of error detection that less than $80 \%$ only on Pattern 3 and Pattern 5. As well as, method 3: sliding window based window size adjusting method presented accuracies of error detection that less than $80 \%$ only on Pattern 3 , Pattern 4 and Pattern 5. 
Table 1. Accuracy of Spelling Error Detection in Basic Vocabulary

\begin{tabular}{|c|c|c|c|c|}
\hline \#Patterns & Patterns of Error & $\begin{array}{c}\text { Method 1: Once } \\
\text { Sliding }\end{array}$ & $\begin{array}{c}\text { Method 2: Initial } \\
\text { Word Fixing }\end{array}$ & $\begin{array}{c}\text { Method 3: Window } \\
\text { Size Adjusting }\end{array}$ \\
\hline 1 & computers & $95.23 \%$ & $85.71 \%$ & $88.88 \%$ \\
\hline 2 & ccomputer & $4.76 \%$ & $85.71 \%$ & $88.88 \%$ \\
\hline 3 & commputer & $33.33 \%$ & $71.42 \%$ & $66.67 \%$ \\
\hline 4 & comtuter & $83.33 \%$ & $83.33 \%$ & $50 \%$ \\
\hline $\mathbf{5}$ & comtter & $\mathbf{4 0 \%}$ & $\mathbf{7 3 . 3 3} \%$ & $\mathbf{3 7 . 5} \%$ \\
\hline 6 & compute & $94.44 \%$ & $94.44 \%$ & $87.5 \%$ \\
\hline 7 & omputer & $0.00 \%$ & $83.33 \%$ & $87.5 \%$ \\
\hline \multicolumn{2}{|r|}{ Average of Accuracy } & $50.16 \%$ & $\mathbf{8 2 . 4 7} \%$ & $72.42 \%$ \\
\hline
\end{tabular}

To conclude, the method 2 presented higher average accuracy of spelling error detection $(82.47 \%)$ than others, especially on Pattern 5, that method 1 and method 3 achieved only $40 \%$ and $37.5 \%$, respectively.

\subsubsection{Correctly Spelled-Words Plus Additional Character}

The misspelled words in this subsection are referred from (Fitzgerald, 1932). Herein, ten frequent misspelled words are selected to evaluate the proposed methods.

Table 2 shows that three proposed methods presented almost equally accuracy of error detection on Word 7 , which are $72.22 \%, 72.22 \%$, and $62.50 \%$, respectively. Otherwise, the highest accuracies of words are performed by method 2 .

Table 2. Accuracy of Spelling Error Detection in Misspelled Words

\begin{tabular}{|c|c|c|c|c|}
\hline \#Word & Misspelled Word & $\begin{array}{c}\text { Method 1: Once } \\
\text { Sliding }\end{array}$ & $\begin{array}{c}\text { Method 2: Initial } \\
\text { Word Fixing }\end{array}$ & $\begin{array}{c}\text { Method 3: Window } \\
\text { Size Adjusting }\end{array}$ \\
\hline 1 & agressive & $25 \%$ & $91.67 \%$ & $80 \%$ \\
\hline $\mathbf{2}$ & maintainance & $\mathbf{4 0 \%}$ & $\mathbf{9 0 \%}$ & $\mathbf{4 1 . 6 7 \%}$ \\
\hline 3 & personell & $76.19 \%$ & $90.47 \%$ & $33.33 \%$ \\
\hline 4 & facinating & $11 \%$ & $85.18 \%$ & $72.72 \%$ \\
\hline $\mathbf{5}$ & restarant & $\mathbf{5 0} \%$ & $\mathbf{8 3 . 3 3} \%$ & $\mathbf{4 0 \%}$ \\
\hline 6 & experiance & $87.50 \%$ & $87.50 \%$ & $30 \%$ \\
\hline 7 & nieghbor & $72.22 \%$ & $72.22 \%$ & $\mathbf{3 7 . 5 0} \%$ \\
\hline $\mathbf{8}$ & orignal & $\mathbf{5 0 \%}$ & $\mathbf{7 7 . 7 8} \%$ & $62.50 \%$ \\
\hline 9 & wellfare & $40 \%$ & $73.33 \%$ & $44.44 \%$ \\
\hline 10 & secratary & $85.71 \%$ & $85.71 \%$ & 50.47 \\
\hline \multicolumn{2}{|c|}{ Average of Accuracy } & $53.76 \%$ & $\mathbf{8 3 . 7 2} \%$ & \\
\hline
\end{tabular}

To conclude, the method 2 still presented higher average accuracy of spelling error detection (83.72\%) than others, especially on Word 2, Word 5, and Word 8, that method 1 and method 3 achieved only 37.50\%-50\%. 


\subsection{Measuring Similar Word in Medical Terms}

The accuracy of similar word measurement in medical terms is focused in this section. Herein, the medical terms: the symptoms of abdomen are collected from the public hospital in Thailand. The spelling errors of symptoms are detected and measured as similar words.

To evaluate the performance for measuring similar words in the medical terms, the proposed sliding window based methods are tested on the regular ten symptoms of abdomen recommended by the medical professionals. The results are shown in Table 3.

Table 3. Accuracy of Similar Word Measurement in Medical Terms

\begin{tabular}{|c|c|c|c|c|c|c|}
\hline \#Word & Word & Similar Word & $\begin{array}{c}\text { Method 1: } \\
\text { Once Sliding }\end{array}$ & $\begin{array}{c}\text { Method 2: } \\
\text { Initial Word } \\
\text { Fixing }\end{array}$ & $\begin{array}{c}\text { Method 3: } \\
\text { Window Size } \\
\text { Adjusting }\end{array}$ & $\begin{array}{c}\text { Replacement } \\
\text { recommended by } \\
\text { Experts }\end{array}$ \\
\hline 1 & Abdomen & Abdominal & $86.67 \%$ & $86.67 \%$ & $11.11 \%$ & $\checkmark$ \\
\hline 2 & Antiemetics & Antinauseant & $33.33 \%$ & $59.25 \%$ & $16.67 \%$ & $\checkmark$ \\
\hline 3 & Backache & Back Pain & $50 \%$ & $72.22 \%$ & $0 \%$ & $\checkmark$ \\
\hline 4 & $\begin{array}{c}\text { Cough, } \\
\text { Chronic }\end{array}$ & Cough, Productive & $50 \%$ & $69.44 \%$ & $5.88 \%$ & $\checkmark$ \\
\hline 5 & $\begin{array}{c}\text { Debility, } \\
\text { General }\end{array}$ & Debilitating Disease & $40 \%$ & $57.78 \%$ & $0 \%$ & $\checkmark$ \\
\hline 6 & Sorensen & Sorethroat & $50 \%$ & $61.11 \%$ & $0 \%$ & $\checkmark$ \\
\hline 7 & $\begin{array}{c}\text { Ankle Foot } \\
\text { Pain }\end{array}$ & Ankle Pain & $38.46 \%$ & $69.33 \%$ & $33.33 \%$ & $\checkmark$ \\
\hline 8 & Arthritis & Arthralgia & $57.14 \%$ & $66.67 \%$ & $0 \%$ & $\checkmark$ \\
\hline 9 & Myocelialgia & Myalgia & $10 \%$ & $60 \%$ & $41.67 \%$ & \\
\hline 10 & $\begin{array}{c}\text { Abdominal } \\
\text { Discomfort }\end{array}$ & $\begin{array}{c}\text { Abdominal } \\
\text { Distention }\end{array}$ & $66.67 \%$ & $75.92 \%$ & $5 \%$ & $\checkmark$ \\
\hline \multicolumn{2}{|c|}{ Average of Accuracy } & $48.23 \%$ & $\mathbf{6 7 . 8 4 \%}$ & $11.37 \%$ & \\
\hline
\end{tabular}

From Table 3, method 2: sliding window based two-word inspection by initial word fixing method showed the highest average accuracy of similar word measurement with $67.84 \%$. To summaries, the method 2 performed excellent spelling error detection, and measuring the similarity among the medical terms. To compare with the experts' recommendation, the words can be replaced by the similar words when the measured values are greater than $50 \%$.

\section{CONCLUSION}

This study presents a method for verifying the similarity of words using the innovative Sliding Windows concept that has been applied in 3 methods. 1. sliding window based two-word inspection by once sliding method 2 . sliding window based two-word inspection by initial word fixing method and 3. sliding window based window size adjusting method. The evaluated is divided into 2 parts, 1) spelling error detection in general words consisting of basic vocabulary and misspelled words retrieved from research, and 2) measuring similar word in medical terms. All parts presented that when applied with subsection basic vocabulary, method 2 presented maximum efficiency and covered all pattern, while method 1 and method 3 could effective in some patterns.

When evaluated in subsection Misspelled Words, it was discovered that the method 2, which was effective from the first subsection, was still effective in evaluating this subsection as before, while method 1 and method 3 had reduced efficiency. And when evaluated in part 2 Measuring Similar Word in Medical Terms, it was discovered that all methods were reduced percentage, method 1 and method 3 decreased to less than $50 \%$, while method 2 slightly decreased accuracy. The average is approximately $70 \%$. All Examination presented that the performance of method 2 is the most effective, even through two forms of testing, and have the capability to group medical terminology. 


\section{REFERENCES}

A. F. Healy., (1981). The effects of visual similarity on proofreading for misspellings. Memory \& Cognition, Vol. 9, No. 5, pp. 453-460.

A. W. Moore and K. Papagiannaki., (2005). Toward the Accurate Identification of Network Applications. Passive and Active Network Measurement, Vol. 3431, pp 41-54.

D. G. Mackay., (1969). The repeated letter effect in the misspellings. Perception \& Psychophysics, Vol. 5, No. 2, pp. 102-106.

Flor, M. (2012). Four types of context for automatic spelling correction. Traitement Automatique des Langues (TAL). 53(3). pp 61-99.

Gammanee, S. and Sodsee, S., (2018). The application of clustering algorithms in screening patients. The Fourteenth National Conference on Computing and Information Technology. Chiang Mai, Thailand, pp. 462-467.

J. A. Fitzgerald., (1932). Words Misspelled most Frequently by Children of the Fourth, Fifth, and Sixth Grade Levels in Life outside the School. The Journal of Educational Research, Vol. 26, No. 3, pp. 213-218.

Köhler, Sven. et al, (2012). Sliding Window Calculations on Streaming Data using the Kepler Scientific Workflow System. Procedia Computer Science. Vol. 9, pp. 1639-1646

Phalasuek, R. et al, (2018). Participatory Action Research: Development of a Participatory Process. The Southern College Network Journal of Nursing and Public Health, Vol. 5, No. 1,pp 211-223.

Sawaengdee, K., (2017). Crisis of Nursing shortage in Health Service Facilities under Office of Permanent Secretary. Journal of Health Science, Vol. 26, No. 2, pp 456-468. 